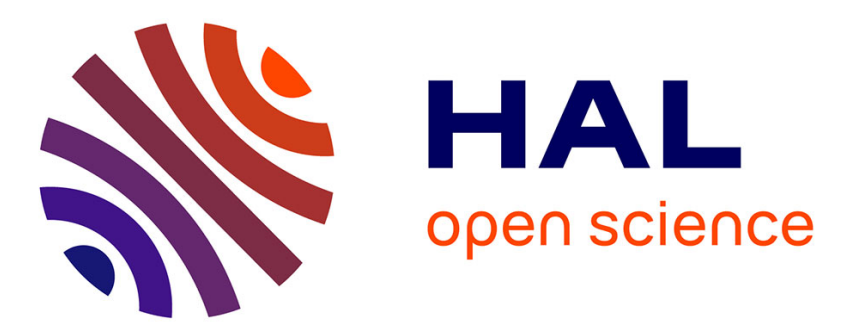

\title{
Recherche de l'origine génétique de plantes à phénotype maternel chez le pommier (Malus x domestica Borkh.). I. - Etude de descendances issues de pollinisation par les abeilles
}

Elisabeth Chevreau, Yves Lespinasse, Marie Godicheau, André Fouillet

\section{- To cite this version:}

Elisabeth Chevreau, Yves Lespinasse, Marie Godicheau, André Fouillet. Recherche de l'origine génétique de plantes à phénotype maternel chez le pommier (Malus x domestica Borkh.). I. - Etude de descendances issues de pollinisation par les abeilles. Agronomie, 1985, 5 (10), pp.905-914. hal00884720

\section{HAL Id: hal-00884720 \\ https://hal.science/hal-00884720}

Submitted on 1 Jan 1985

HAL is a multi-disciplinary open access archive for the deposit and dissemination of scientific research documents, whether they are published or not. The documents may come from teaching and research institutions in France or abroad, or from public or private research centers.
L'archive ouverte pluridisciplinaire HAL, est destinée au dépôt et à la diffusion de documents scientifiques de niveau recherche, publiés ou non, émanant des établissements d'enseignement et de recherche français ou étrangers, des laboratoires publics ou privés. 


\title{
Recherche de l'origine génétique de plantes à phénotype maternel chez le pommier (Malus $\times$ domestica Borkh.). I. - Etude de descendances issues de pollinisation par les abeilles
}

\author{
Elisabeth CHEVREAU, Yves LESPINASSE, Marie GODICHEAU \& André FOUILLET \\ I.N.R.A., Statiun d'Arboriculture fruitière, Beaucouzé, F 49000 Angers
}

RÉSUMÉ

\begin{abstract}
Plusieurs variétés de pommier ont été pollinisées par un clone porteur d'un gène marqueur de coloration rouge, homozygote dominant, dans le but de détecter des haplö̈des spontanés. Parmi les descendants, un faible taux de plantes à phénotype vert, donc maternel, est apparu ; un seul haploïde a été identifié. L'origine génétique des descendants à phénotype maternel diplö̈des et triploïdes est étudiée par l'observation de plusieurs caractères agronomiques et l'analyse des isozymes du pollen. Les résultats démontrent l'apport de pollen étranger par les abeilles employées pour effectuer la pollinisation; ils indiquent aussi le rôle probable de l'autofécondation pour l'obtention de descendants diploïdes chétifs ou triplö̈des. L'hypothèse du développement par parthénogenèse d'oosphères diploïdes peut aussi être avancée. D'autres résultats portant sur des croisements récents et utilisant différents modes de pollinisation seront présentés dans un $2^{\mathrm{e}}$ article.
\end{abstract}

Mots clés additionnels : Gène marqueur, autofécondation, parthénogenèse, haplö̈de, isozymes, caractères quantitatifs.

Study of the genetic origin of plants with a maternal phenotype in apple (Malus $\times$ domestica Borkh.). I. - Progenies obtained from pollination by bees.

Several varieties were pollinated by a selected clone carrying a homozygous dominant marker gene for red colour, in order to detect spontaneous haploids. Among the progenies, green seedlings, presumably of maternal origin, appeared at a low rate ; only one haploid plant was identified. The genetic origin of the green diploid and triploid seedlings was studied by means of pollen isozyme analysis and morphological and agronomic characters. Some of the plants proved to be out-crosses due to alien pollen carried by the honeybees used for pollination ; results indicated also that triploid and very stunted diploid plants probably arose from selfing. Parthenogenetic development of diploid egg-cells could also be postulated. Further results obtained from recent crosses involving different kinds of pollination will be presented in a second paper.

Additional key words : Marker gene, selfing, parthenogenesis, haploid, isozymes, quantitative characters.

\section{INTRODUCTION}

La recherche d'haploïdes spontanés a été entreprise dans l'espèce pommier (Malus $\times$ domestica Borkh.), à l'aide d'une technique de marquage génétique. Les objectifs et la méthode utilisée ont déjà été décrits (LESPINASSE \& GODICHEAU, 1980). Plusieurs variétés ont été pollinisées par un clone porteur d'un gène dominant $\mathrm{R}$, responsable de la couleur rouge de tous les organes, à l'état homozygote. La majorité des descendants obtenus est à phénotype rouge, donc de nature hybride. Un faible taux de plantes à phénotype vert est également apparu. Parmi elles, une plante haploïde a été identifiée ; elle provient certainement du développement d'une des cellules du sac embryon- naire haploïde. Le but de cette étude est d'analyser l'origine génétique des individus diploïdes ou triploïdes, à phénotype maternel, issus de ce programme de croisements.

Plusieurs hypothèses peuvent être proposées ; selon le mécanisme en cause, les caractéristiques génétiques des descendants sont bien différentes:

- L'apport accidentel de pollen étranger est la $1^{\text {re }}$ hypothèse à envisager.

- L'apomixie par aposporie existe chez plusieurs espèces du genre Malus (SAX, 1959; SCHMIDT, 1977) ; elle a également été observée chez certaines variétés cultivées de pommier (SHERMAN et al., 1978). Les individus issus d'un tel mécanisme posșèdent exactement le génotype de la plante mère. 
- Un système d'auto-incompatibilité du type gamétophytique existe chez le pommier. Cependant il s'exprime à des degrés différents selon les variétés : un faible taux de fructification accompagné d'un nombre réduit de pépins par fruit peut être obtenu en autofécondant des variétés partiellement auto-compatibles comme «Golden Delicious ». De plus, des températures basses $\left(15^{\circ} \mathrm{C}\right)$ lors de la pollinisation peuvent diminuer l'expression de l'auto-incompatibilité, en autorisant une croissance plus longue des tubes polliniques incompatibles (MODLIBOWSKA, 1945). Enfin, la possibilité d'autofécondation par des grains de pollen diploïdes a été démontrée dès 1942 par LEWIS \& MODLIBOWSKA, en autofécondant des clones tétraploïdisés de variétés de poirier ; elle a été vérifiée sur le pommier par KAPUSTA (1983). L'interprétation proposée est la suivante: dans un grain de pollen diploïde, hétéro-allélique pour le locus $\mathrm{S}$ d'incompatibilité, l'interaction entre les 2 allèles conduit à un "affaiblissement mutuel» qui supprime la réaction d'auto-incompatibilité. On peut donc supposer que certains des individus triploïdes à phénotype maternel sont issus d'autofécondation par un grain de pollen non réduit.

- Le contrôle du phénotype rouge est attribué à un seul gène dominant $R$ (DECOURTYE, 1967). Cependant, il existe parmi les plantes à phénotype rouge d'importantes différences d'intensité de la coloration des feuilles et des fruits. Or, le pommier a pour nombre de base $\mathrm{x}=17$ chromosomes et est considéré comme un polyploïde secondaire d'origine auto ou allopolyploïde; l'analyse enzymatique a fourni des arguments en faveur de l'hypothèse d'allopolyploïdie (CHEvreau, 1984). Prenant en compte l'hypothèse d'une origine autopolyploïde, ZwINTSCHER (1973) attribue le contrôle du phénotype rouge à 3 loci homologues et explique les variations d'intensité par les différences de dosage allélique. Si l'on admet que le gène marqueur $R$ est en fait constitué de plusieurs loci homologues ou homéologues, des gamètes recombinés peuvent conduire à la formation d'hybrides à phénotype vert.

- Le développement parthénogénétique d'oosphères diploïdes issues de restitution à la méïose peut également être envisagé. La formation de gamètes de ce type est un phénomène connu chez différentes plantes, en particulier la pomme de terre. MOK \& PELOQUIN (1975a) ont mis en évidence, dans des clones diploïdes de cette espèce, 2 types de mécanismes conduisant à la formation de gamètes mâles non réduits :

- l'orientation parallèle des fuseaux à l'anaphase II, au lieu de l'orientation normale à $60^{\circ}$, aboutit à la formation d'une diade au lieu d'une tétrade. C'est un cas de restitution à la $1^{\text {re }}$ division (F.D.R.),

- une cytokinèse précoce, juste après la $1^{\text {re }}$ division méiotique, et l'absence d'une $2^{\mathrm{e}}$ division conduit également à une diade, par restitution à la $2^{\mathrm{e}}$ division (S.D.R.).

Les conséquences génétiques sont bien différentes dans les 2 cas. Par F.D.R., l'hétérozygotie est transmise aux gamètes à $100 \mathrm{p}$. 100 pour tous les loci entre le centromère et le $1^{\text {er }}$ crossing-over, et à 50 p. 100 pour ceux situés entre le $1^{\mathrm{er}}$ et le $2^{\mathrm{e}}$ crossing-over. Par S.D.R., les loci hétérozygotes chez le parent ne seront hétérozygotes dans les gamètes que s'ils sont situés entre le $1^{\text {er }}$ et le $2^{\text {e }}$ crossing-over. Les 2 phénomènes ont également été détectés lors de la gamétogenèse femelle (TAYLOR, 1978).

- Le développement par parthénogenèse d'oosphères ayant subi un doublement post-méïotique est une autre interprétation. Ceci conduirait à la formation d'individus identiques à des haploïdes doublés.

L'analyse des plantes à phénotype maternel a été conduite selon plusieurs approches complémentaires : comptages chromosomiques, observation de différents caractères agronomiques des arbres et des fruits, analyse par électrophorèse des isozymes du pollen ou des feuilles. L'ensemble de ce programme a fourni 55000 semis, dont 1218 étaient verts. Dans cette $1^{\text {re }}$ partie, seule l'étude des descendances les plus anciennes, issues de pollinisation par les abeilles, est rapportée. L'analyse des descendances les plus récentes, issues de différents modes de pollinisation, sera présentée dans une $2^{\mathrm{e}}$ partie.

\section{MATÉRIEL ET MÉTHODES}

\section{A. Les pollinisations effectuées}

Le parent mâle de tous les croisements effectués est un clone appelé T.N.R., porteur du gène marqueur $R$ à l'état homozygote. Cet individu est issu, en $2^{\mathrm{e}}$ génération du croisement : Malus niedzwetzkyana $\times$ Malus domestica cv. "Jonathan ».

Plusieurs variétés ont été utilisées comme parent femelle : en 1975, «Golden Delicious », appelée par la suite «Golden », et, en 1976, 3 mutants ayant pour origine la variété «Delicious »: «Topred ", " Redspur », "Wellspur ». Avant la floraison, les arbres sont isolés sous une cage étanche au pollen. La pollinisation est effectuée en introduisant sous la cage une ruchette d'abeilles, prélevée le matin chez un apiculteur, et un arbre en pot du pollinisateur T.N.R., en pleine floraison. Cette méthode a été adoptée, car elle réduit considérablement les opérations de pollinisation.

Le repérage des descendants verts peut s'effectuer en serre, dès les $1^{\text {res }}$ semaines après le semis.

\section{B. Les observations cytologiques}

Un comptage chromosomique a été réalisé systématiquement sur tous les individus verts à phénotype chétif, potentiellement haploïdes, ainsi que sur la plupart des individus verts à phénotype normal. L'observation de cellules en mitose a été réalisée sur pointes de racines, sur de jeunes semis en croissance d'environ 2 mois, selon la méthode décrite par LESPINASSE (1973).

\section{Les observations sur arbres adultes}

Onze caractères ont été observés au champ ; le type de notation adopté ici est celui employé pour le jugement des hybrides en cours de sélection :

- la vigueur des arbres sur leurs propres racines est appréciée par la mesure du tour de tronc à $20 \mathrm{~cm}$ au-dessus du sol, exprimé en $\mathrm{mm}$; 
- la date de floraison correspond au stade où 50 p. 100 des corymbes de l'arbre sont en F1, selon l'échelle établie par FLECKINGER (1964). Elle est exprimée en nombre de jours à partir du 10 avril ;

- la date de récolte, appréciée par la dégustation des fruits, est exprimée en nombre de jours à partir du 10 août ;

- la couleur des fruits a été notée par une échelle de 7 classes allant du vert (classe 1) au rouge foncé (classe 7) ;

- la sensibilité des fruits à 4 types d'accidents physiologiques a été notée ; la maladie des taches liégeuses ou bitter-pit, la vitrescence, la présence de rugosité ou de craquelures déprécient l'aspect et la qualité des fruits. Une échelle de 4 classes de sensibilité a été employée pour chaque caractère, allant de 1 pour les arbres indemnes à 4 pour les individus très atteints. Ces 4 notes ont ensuite été intégrées dans un " indice global de sensibilité », qui peut prendre des valeurs entre 0 , pour les individus très senșibles, et 100 pour ceux qui ne présentent aucun de ces défauts ;

- 3 critères ont été pris en compte pour estimer la valeur gustative des fruits : la teneur en jus, la texture et la saveur des fruits ont été notés selon une échelle allant de 1 (très mauvaise qualité) à 9 (qualité excellente). Là aussi, ces 3 notes ont été regroupées dans un " indice global de qualité gustative » variant de 0 à 100.

L'ensemble de ces observations a été réalisé sur les individus à phénotype maternel issus de 2 croisements, ainsi que sur 2 descendances témoins : l'une constituée d'individus rouges, donc hybrides, issue du même programme de croisement, l'autre issue de l'autofécondation du clone P7 R34 A20, lui-même ayant été obtenu par autofécondation de la variété «Golden Delicious ».

\section{Les analyses isoenzymatiques}

Les 4 descendances ayant fait l'objet d'observations morphologiques ont également été analysées par électrophorèse, à partir d'extraits de pollen. Huit systèmes enzymatiques ont été étudiés : estérase (EST), phosphatase acide (AP), endopeptidase (ENP), leucine-amino-peptidase (LAP), alcool déshydrogénase $(\mathrm{ADH})$, isocitrate déshydrogénase (IDH), phosphoglu- comutase (PGM) et phosphoglucoisomérase (PGI). Les analyses ont été réalisées en gel d'acrylamide ou d'amidon. La technique employée a déjà été décrite (Chevreau, 1984)

\section{RÉSULTATS}

\section{A. Effectifs obtenus et niveau de ploïdie}

Ces résultats figurent au tableau 1. Le nombre de base de cette espèce est de $\mathrm{x}=17$ chromosomes $(2 n=34)$. On constate un écart important entre les taux de plantes vertes obtenus en 1975 avec la variété «Golden» et en 1976 avec les 3 mutants issus de "Delicious ». Cette différence peut être attribuée à 2 types d'effets :

- les conditions climatiques de l'année, lors de la pollinisation : l'année 1975 fut marquée par des températures particulièrement élevées lors de cette période ;

- l'effet dû au génotype maternel ; les 3 mutants "Wellspur »; « Redspur » et «Topred» ont certainement un génotype extrêmement voisin ; ils n'ont aucune parenté avec la variété «Golden ».

Une seule plante haploïde a été isolée parmi 9969 semis. Les comptages chromosomiques effectués sur un échantillon des 4 descendances révèlent une proportion de triplö̈des variant de 4 à 12 p. 100 . Ce taux est élevé ; en effet, la fréquence de triploïdes décelée par EINSET (1952) après examen de 6825 plantules issues de croisements entre différentes variétés diploïdes est seulement de 0,28 p. 100 .

\section{B. Les observations sur arbres adultes}

Dans les 2 descendances d'individus verts et les 2 descendances témoins étudiées, tous les individus portant des fruits en 1982 ont été observés. Les effectifs sont les suivants :

- 69 descendants verts du croisement « Golden 》 $\times$ «T.N.R. », dont 9 triploïdes, désignés par le terme « descendance verte de Golden »;

- 73 descendants verts du croisement «Topred» $\times$ «T.N.R. », désignés par le terme « descendance verte de Topred »;

TABLEAU 1

Effectif et niveau de ploidie des descendants à phénotype maternel.

Total number and ploidy level of seedlings with a maternal phenotype.

\begin{tabular}{|c|c|c|c|c|c|c|c|c|}
\hline \multirow{2}{*}{ Parent femelle } & \multirow{2}{*}{$\begin{array}{l}\text { Nombre total } \\
\text { de semis }\end{array}$} & \multirow{2}{*}{\multicolumn{2}{|c|}{$\begin{array}{c}\text { Nombre et } \% \\
\text { de plantules vertes }\end{array}$}} & \multirow{2}{*}{$\begin{array}{l}\text { Nombre de plantules } \\
\text { vertes étudiées }\end{array}$} & \multicolumn{4}{|c|}{ Nombre de plantules à } \\
\hline & & & & & $17 \mathrm{chr}^{*}$ & $34 \mathrm{chr}$ & $35 \mathrm{chr}$ & $51 \mathrm{chr}$ \\
\hline Golden & 4552 & 116 & 2,5 & 116 & - & 102 & - & $14(12,1 \%)$ \\
\hline Topred & 1243 & 122 & 9,8 & 107 & 1 & 100 & 3 & 3 \\
\hline Redspur & 1696 & 135 & 8,0 & 117 & - & 106 & 2 & 9 \\
\hline Wellspur & 2478 & 262 & 10,6 & 93 & - & 85 & 5 & 3 \\
\hline Total des types « Delicious » & 5417 & 519 & 9,6 & 317 & 1 & 291 & 10 & $15(4,7 \%)$ \\
\hline
\end{tabular}

* chr : chromosomes. 
- 58 descendants rouges du croisement «Topred » $\times$ «T.N.R. ».

- 99 descendants d'autofécondation de P7 R34 A20.

Les distributions observées pour chaque caractère sont décrites par leurs histogrammes (fig. 1 à fig. 6) leurs moyennes et leurs variances (tabl. 2). La condition de normalité n'étant pas satisfaite dans la plupart des cas, aucun test de comparaison de ces moyennes et variances n'a pu être appliqué. Pour apprécier les différences entre distributions, un test non paramétrique a été utilisé : deux à deux, les distributions observées sont comparées à leur distribution cumulée, par la statistique « 2 I », qui suit approximativement une loi de $\chi^{2}$ (SOKAL \& ROHLF, 1969).

Les caractéristiques des parents des descendances sont rassemblées dans le tableau 3 .

\section{Accidents physiologiques}

Le niveau de sensibilité au bitter-pit, aux craquelures et à la vitrescence est très bas dans toutes les descendances; les parents de ces croisements en sont d'ailleurs tous indemnes. La sensibilité à la rugosité est plus variable : la descendance d'autofécondation et la " descendance verte de Golden » sont caractérisées par une note moyenne de sensibilité élevée. Les

TABLEAU 2

Moyennes et variances des distributions des 4 descendances observées.

Means and variances of the 4 observed progenies.

\begin{tabular}{|c|c|c|c|c|c|}
\hline & & $\begin{array}{c}\text { Rouges } \\
\text { «Topred } » \times \ll \text { T.N.R. » }\end{array}$ & $\begin{array}{c}\text { Verts } \\
\text { «Topred } » \times \ll \text { T.N.R. » }\end{array}$ & $\begin{array}{c}\text { Verts } \\
« \text { Golden } » \times \ll \text { T.N.R. » }\end{array}$ & $\begin{array}{l}\text { Autofécondation } \\
\text { P7 R34 A20 }\end{array}$ \\
\hline $\begin{array}{l}\text { Indice global } \\
\text { de sensibilité }\end{array}$ & $\begin{array}{l}\text { Moyenne } \\
\text { Variance }\end{array}$ & $\begin{array}{r}89,18 \\
362\end{array}$ & $\begin{array}{r}76,26 \\
532\end{array}$ & $\begin{array}{r}64,42 \\
847\end{array}$ & $\begin{array}{r}71,84 \\
620\end{array}$ \\
\hline Couleur du fruit & Variance & 0,24 & 2,93 & 2,79 & 0,43 \\
\hline $\begin{array}{l}\text { Indice global } \\
\text { de qualité }\end{array}$ & $\begin{array}{l}\text { Moyenne } \\
\text { Variance }\end{array}$ & $\begin{array}{r}17,64 \\
281\end{array}$ & $\begin{array}{r}27,62 \\
406\end{array}$ & $\begin{array}{r}33,42 \\
468\end{array}$ & $\begin{array}{r}48,43 \\
321\end{array}$ \\
\hline Date de floraison & $\begin{array}{l}\text { Moyenne } \\
\text { Variance }\end{array}$ & $\begin{array}{r}19,65 \\
11\end{array}$ & $\begin{array}{r}20,06 \\
10\end{array}$ & $\begin{array}{c}21,13 \\
14\end{array}$ & $\begin{array}{r}14,44 \\
8\end{array}$ \\
\hline Date de récolte & $\begin{array}{l}\text { Moyenne } \\
\text { Variance }\end{array}$ & $\begin{array}{r}54,06 \\
219\end{array}$ & $\begin{array}{r}51,79 \\
335\end{array}$ & $\begin{array}{r}57,05 \\
482\end{array}$ & $\begin{array}{r}51,22 \\
78\end{array}$ \\
\hline Vigueur & $\begin{array}{l}\text { Moyenne } \\
\text { Variance }\end{array}$ & $\begin{array}{r}180 \\
1072\end{array}$ & $\begin{array}{r}173 \\
1671\end{array}$ & $\begin{array}{r}154 \\
2673\end{array}$ & $\begin{array}{r}176 \\
1473\end{array}$ \\
\hline
\end{tabular}

TABLEAU 3

Caractéristiques des parents des descendances observées.

Characteristics of the parents of the observed progenies.

\begin{tabular}{|c|c|c|c|c|}
\hline & Golden & P7 R34 A20 & Topred & T.N.R. \\
\hline Vitrescence & 1 & 1 & 1 & 1 \\
\hline Bitter-pit & 1 & 1 & 1 & 1 \\
\hline Craquelures & $i$ & 1 & 1 & 1 \\
\hline Rugosité & 3 & 3 & 2 & 4 \\
\hline Indice global de sensibilité & 80 & 80 & 97,5 & 50 \\
\hline Couleur & 3 & 2 & 6 & 7 \\
\hline Jus & 5 & 7 & 4 & 4 \\
\hline Texture & 4 & 4 & 4 & 2 \\
\hline Saveur & 7 & 5 & 4 & 2 \\
\hline Indice global de qualité & 56,6 & 56,6 & 20 & 6,6 \\
\hline Date de $\mathrm{F} 1^{*}$ & 14 & 14 & 12 & 8 \\
\hline Date de récolte** & 50 & 58 & 62 & 70 \\
\hline
\end{tabular}

* En nombre de jours après le 10 avril.

** En nombre de jours après le 10 août. 


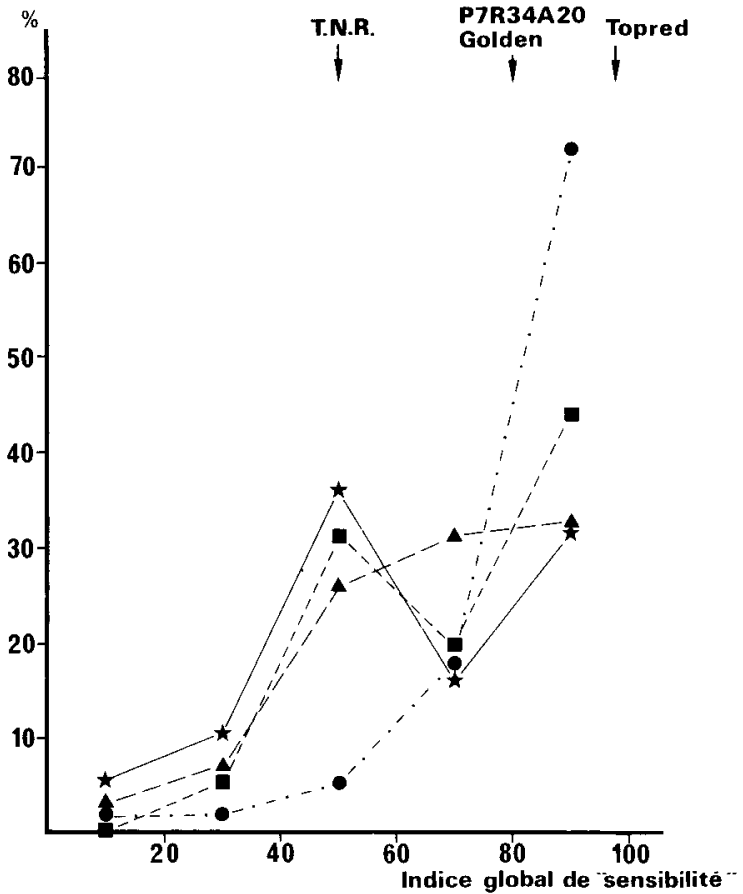

Figure 1

Histogrammes des distributions de "l'indice global de sensibilité ». "Global susceptibility index": histograms.

- .-.- - rouges - red plants - "Topred 》 $\times$ «T.N.R. 》. - ... - verts - green plants - «Topred» $\times$ 《T.N.R.». $\star-\star$ verts - green plants - «Golden » $\times$ «T.N.R. ».

A - - $\mathbf{A}$ autofécondation - selfing - P7 R34 A20.

distributions de l'indice global de sensibilité sont données figure 1 . D'après le test « $2 \mathrm{I}$ », seule la descendance d'hybrides rouges est significativement différente des 3 autres, au seuil de 5 p. 100 ; la majorité de ces individus a un indice global élevé, indiquant un bon comportement vis-à-vis des 4 types d'accidents physiologiques. La variance la plus élevée est celle de la "descendance verte de Golden"; ceci peut être attribué à son hétérogénéité de sensibilité à la rugosité.

\section{Couleur des fruits}

Les histogrammes des distributions sont donnés figure 2. Les moyennes ne sont pas mentionnées : elles n'ont aucun sens dans le cas d'une telle variable. La descendance d'autofécondation ne contient que des arbres à fruits verts ou jaunes, comme le parent P7 R34 A20. La descendance hybride entre «Topred» et «T.N.R. » ne contient que des individus à fruits rouges, ce caractère étant sous le contrôle du gène marqueur R transmis par «T.N.R.». Seules les 2 « descendances vertes » sont nettement hétérogènes; on remarque que 40 p. 100 des descendants de "Golden " ont des fruits rouges et 40 p. 100 des descendants de «Topred» des fruits verts ou jaunes.

\section{Qualité gustative des fruits}

Les histogrammes des distributions de l'indice global de qualité sont donnés figure 3. La descendance d'hybrides rouges a la moyenne et la variance les plus faibles; le test « 2 I » indique une différence significative au seuil de 5 p. 100 avec la descendance d'autofécondation et la "descendance verte de Golden».

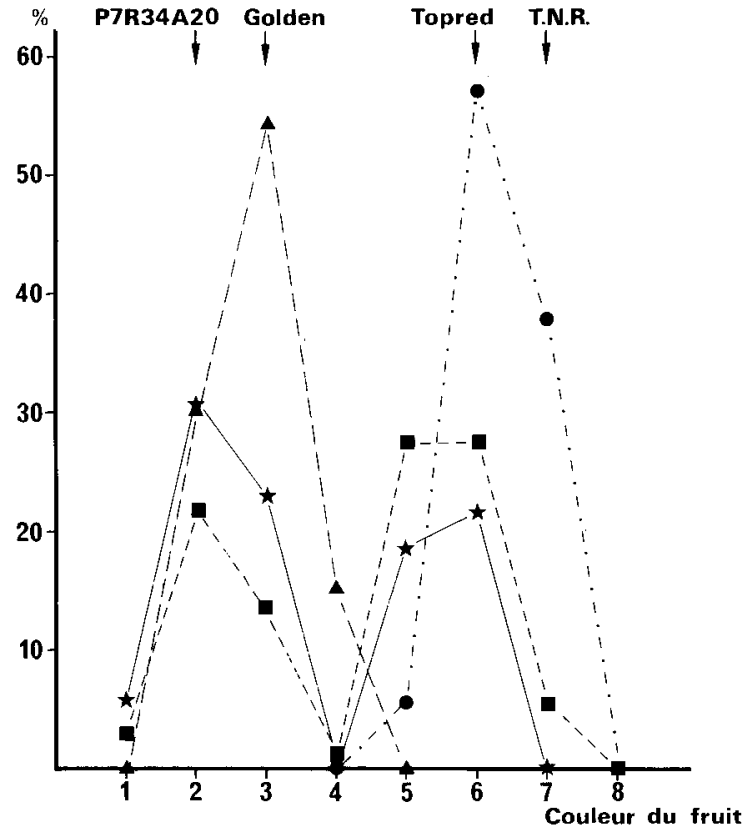

Figure 2

Histogrammes des distributions de la couleur du fruit.

Fruit color: histograms.

- ...- - rouges - red plants - «Topred》 $\times$ «T.N.R.».

- . - - verts - green plants - «Topred » $\times$ «T.N.R.».

$\star$ « verts - green plants - « Golden 》 $\times$ «T.N.R. ».

$\Delta-\ldots+\Delta$ autofécondation - selfing - P7 R34 A20.

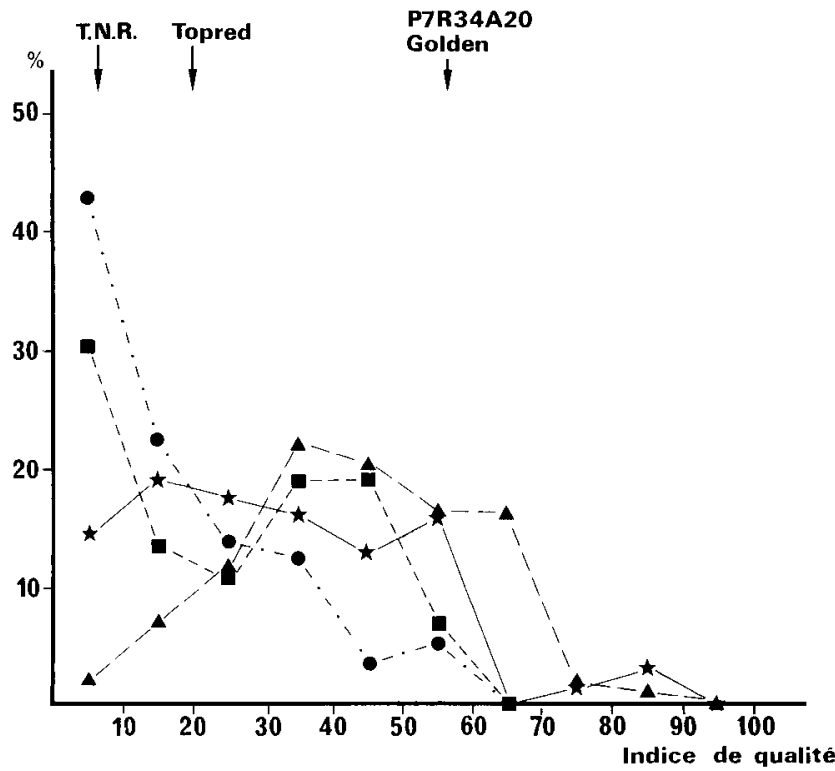

Figure 3

Histogrammes des distributions de "l'indice global de qualité ».

"Global quality index" : histograms.

- .-.- rouges - red plants - "Topred " $\times$ «T.N.R. ".

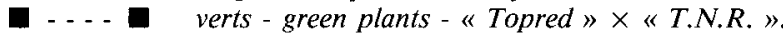

$\star-\star$ verts - green plants - «Golden » $\times$ «T.N.R. ».

$\Delta-\ldots \Delta$ autofécondation - selfing - P7 R34 A20.

Une proportion importante de ces individus ont un indice global très bas.

Parmi les 3 critères d'appréciation, la texture et la saveur sont particulièrement mauvaises dans l'ensemble de cette descendance, tout comme chez le parent mâle «T.N.R. ». La descendance d'autofécondation, 
significativement différente des 3 autres, a la moyenne la plus élevée ; ceci correspond à une teneur en jus élevée et à une bonne saveur moyenne, à l'image de la qualité du parent P7 R34 A20. Les 2 «descendances vertes " ne sont pas significativement différentes. Elles se distinguent des 2 autres par leur plus grande hétérogénéité, en particulier pour le caractère saveur. On note la présence de quelques individus de très bonne qualité dans la "descendance verte de Golden ». L'écart entre les moyennes de ces 2 descendances est en accord avec la différence de qualité entre les parents maternels des 2 croisements.

\section{Date de floraison et de récolte}

Les caractéristiques des distributions de ces caractères sont présentées figures 4 et 5 . Les dates de floraison sont très regroupées ; d'après le test « 2 I ", seule la descendance d'autofécondation diffère significativement des 3 autres, au seuil de 5 p. 100. Ceci est dû à une date de floraison moyenne légèrement plus précoce et à un étalement un peu plus important de la date de floraison. Les distributions des dates de récolte sont toutes significativement différentes 2 à 2 au seuil de 5 p. 100 . La descendance d'autofécondation est particulièrement homogène, alors que la « descendance verte de Golden » est la plus dispersée. Les 2 autres distributions sont nettement bimodales.

\section{Vigueur}

Pour tous les caractères précédents, seuls les arbres en fructification ont pu être notés. Pour apprécier la vigueur d'une descendance, il nous a paru important de prendre en compte tous les individus sans exception ; en effet, ce sont généralement les arbres les plus chétifs qui n'atteignent pas le stade de mise à fruits. Les effectifs observés pour ce caractère sont donc les suivants :

- «descendance verte de Golden »: 108,

- «descendance verte de Topred »: 113,

- descendance d'hybrides rouges : 76,

- descendance d'autofécondation : 110.

Les histogrammes des distributions regroupées par classes de $20 \mathrm{~mm}$ sont donnés figure 6 . La descendance d'autofécondation étant nettement plus âgée (plantée en 1972), elle ne peut être directement comparée aux 3 autres descendances (plantées en 1977 et 1978). Le test « 2 I » indique une différence significative entre les distributions de la « descendance verte de Golden » et de la descendance d'hybrides rouges, ainsi qu'entre les 2 «descendances vertes». La variance la plus faible est celle de la descendance d'hybrides rouges; elle se caractérise par l'absence d'individus d'un tour de tronc inférieur à $80 \mathrm{~mm}$. La « descendance verte de Golden » est extrêmement hétérogène, avec un étalement important de la distribution vers les valeurs faibles. Ce phénomène est moins marqué dans le cas de la «descendance verte de Topred ».

La moyenne de la distribution de la descendance d'autofécondation n'est pas plus élevée que celle des descendances plus jeunes; de plus, le volume total des arbres reste faible. Cette réduction de vigueur s'accompagne souvent de perturbations du port de l'arbre (port étalé, sans axe vertical). Parmi les 2 "descendances vertes", on distingue un groupe

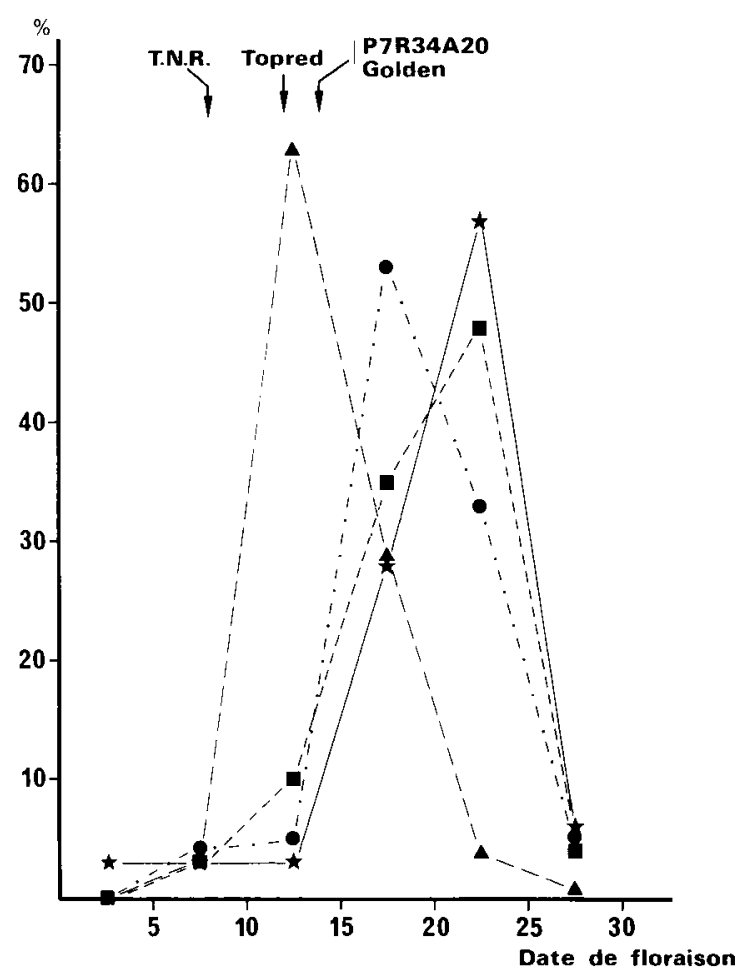

Figure 4

Histogrammes des distributions de la date de floraison. Flowering date : histograms.

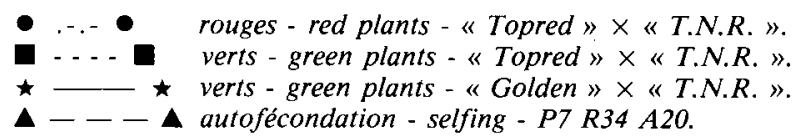

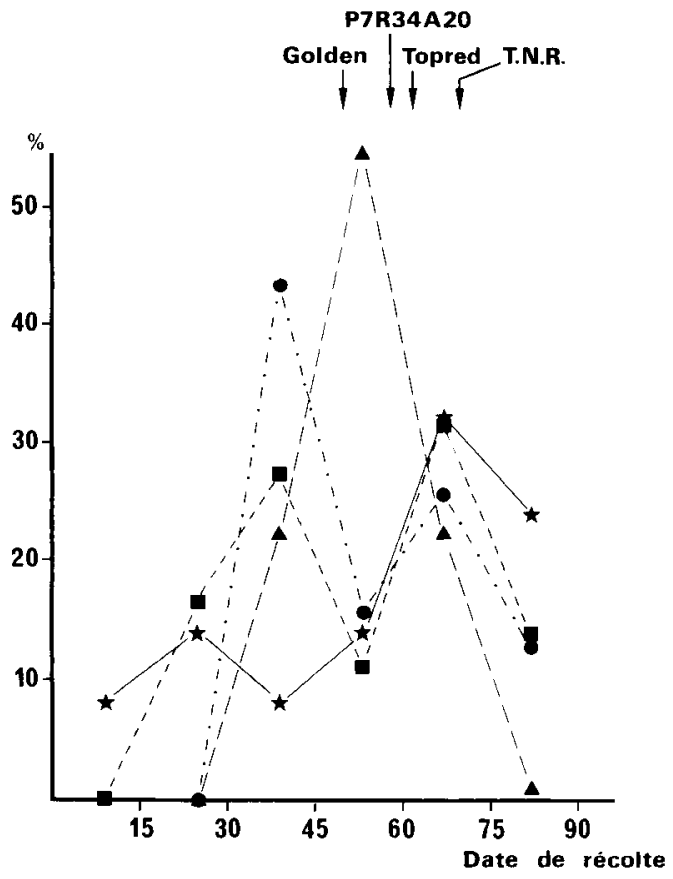

Figure 5

Histogrammes des distributions de la date de récolte.

Harvesting date : histograms.

-..- - rouges - red plants - «Topred" $\times$ «T.N.R. ».

- .. - verts - green plants - "Topred» $x$ «T.N.R.».

$\star-\star$ verts - green plants - « Golden " $\times$ «T.N.R. ».

$\Delta-\ldots-\Delta$ autofécondation - selfing - P7 R34 A20. 


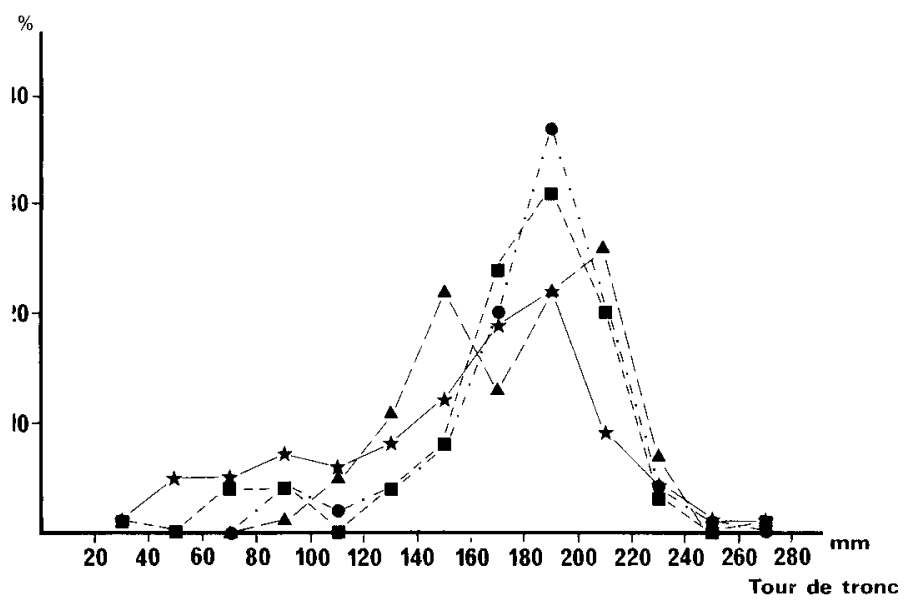

Figure 6

Histogrammes des distributions pour le caractère vigueur.

Vigour : histograms.

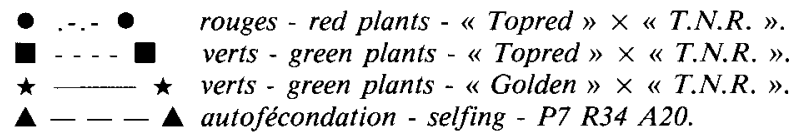

d'individus à l'aspect particulièrement chétif, rappelant le phénotype de certains descendants d'autofécondation. Ils correspondent aux classes de tour de tronc inférieur à $100 \mathrm{~mm}$. Cette notation a également été effectuée parmi les «descendances vertes de Wellspur » et de «Redspur »; les résultats figurent au tableau 4.

\section{Les analyses enzymatiques}

L'analyse de 8 systèmes enzymatiques a été réalisée sur 43 individus de la " descendance verte de Golden $»$, dont 8 triploïdes, et 41 individus de la « descendance verte de Topred». La descendance d'hybrides rouges avait également été analysée dans le cadre d'une étude de déterminisme génétique (CHEVREAU et al., 1986); celle-ci a permis d'établir les relations d'allélisme entre les bandes des diagrammes ainsi que les génotypes des parents. La composition allélique des parents et des $\mathbf{3}$ descendances analysées figure au tableau 5 . On constate la présence, parmi les descendants verts, d'allèles inexistants chez les 2 parents du croisement ; c'est le cas de l'allèle Idh $^{\mathrm{a}}$ dans la « descendance verte de Golden » et des allèles
Enp $^{\mathrm{x}}$, Pgi ${ }^{\mathrm{a}}$ et Lap $^{\mathrm{d}}$ dans celle de «Topred». La comparaison des fréquences alléliques parmi les descendants verts et rouges du croisement «Topred » $\times$ 《T.N.R. » permet de faire plusieurs remarques :

- l'allèle $A p^{a}$, présent chez le parent mâle seulement, est beaucoup plus fréquent parmi les descendants verts ;

- inversement, les allèles $\mathrm{Pgm}^{\mathrm{b}}$ et $\mathrm{Lap}^{\mathrm{b}}$, seulement présents chez «T.N.R. », sont pratiquement absents chez les descendants verts alors qu'ils sont transmis aux descendants rouges dans des proportions voisines de $3 / 4$ et $1 / 2$ respectivement.

Une telle comparaison n'a pu être faite pour la « descendance verte de Golden ». On note cependant que les allèles $\operatorname{Pgm}^{\mathrm{b}}$ et $\mathrm{Lap}^{\mathrm{b}}$, présents chez «T.N.R. », sont totalement absents dans cette descendance.

A l'intérieur de chacune des « descendances vertes》 étudiées, les individus peuvent être classés en 3 groupes (cf. tabl. 6) :

- le groupe 1 comprend tous les individus ne possédant que des allèles présents chez le parent femelle du croisement. Il est important de préciser que la plupart de ces allèles ne sont pas caractéristiques des variétés «Golden » et "Topred », mais présents chez de nombreuses variétés de pommier ;

- le groupe 2 rassemble tous les individus possédant, en plus des allèles présents chez le parent femelle, des allèles présents seulement chez le parent mâle «T.N.R. ». Ici également, on doit noter que ces allèles ne sont pas tous spécifiques du clone «T.N.R. » ;

- le groupe 3 contient les individus possédant au moins un allèle inexistant chez les 2 parents du croisement.

Aucune relation étroite entre l'appartenance à l'un de ces groupes enzymatiques et les caractères des fruits n'a été décelée. Par contre, 8 triploïdes de la descendance de «Golden » ont pu être analysés : ils appartiennent tous au groupe 1 .

\section{DISCUSSION}

Les résultats obtenus apportent un certain nombre de réponses à la question de l'origine des individus à phénotype maternel. L'hypothèse d'une origine apomictique peut être éliminée. En effet, il existe une

TABLEAU 4

Proportion d'individus chétifs parmi les « descendances vertes ».

Proportion of stunted phenotypes among the "green progenies".

Parent femelle

Effectif total de la

Nombre et pourcentage d'individus chétifs

" descendance verte" observée

\begin{tabular}{|c|c|c|c|}
\hline Golden & 108 & 21 , dont $1(3 n)$ & 19,4 \\
\hline Topred & 113 & 10 , dont $3(3 n)$ & 8,8 \\
\hline Redspur & 102 & 20 , dont $3(3 n)$ & 19,6 \\
\hline Wellspur & 249 & 22 , dont $3(3 n)$ & 8,8 \\
\hline Total des types «Delicious» & 464 & 52 & 11,2 \\
\hline
\end{tabular}


TABLEAU 5

Composition allélique des parents et de leurs descendances, pour 8 systèmes enzymatiques. Allelic composition of parents and progenies, for 8 enzymes.

\begin{tabular}{|c|c|c|c|c|c|c|}
\hline \multirow[b]{2}{*}{ Allèles } & \multicolumn{3}{|c|}{ Présence chez: } & \multicolumn{3}{|c|}{ Fréquence $(\%)$ parmi les : } \\
\hline & Golden & Topred & T.N.R. & $\begin{array}{c}\text { Verts } \\
\text { Golden } \times \text { T.N.R. }\end{array}$ & $\begin{array}{c}\text { Verts } \\
\text { Topred } \times \text { T.N.R. }\end{array}$ & $\begin{array}{c}\text { Rouges } \\
\text { Topred } \times \text { T.N.R. }\end{array}$ \\
\hline Enp $^{x}$ & -. & - & - & 0 & 3 & 0 \\
\hline Enp $p^{a}$ & + & + & + & 100 & 97 & 100 \\
\hline Enp ${ }^{b}$ & - & + & + & 77 & 100 & 100 \\
\hline $\mathrm{Ap}^{\mathrm{a}}$ & + & - & + & 73 & 45 & 16 \\
\hline $\mathrm{Ap}^{\mathrm{b}}$ & + & + & + & 100 & 100 & 100 \\
\hline $\operatorname{Pgm}^{\mathrm{a}}$ & + & + & + & 100 & 100 & 100 \\
\hline $\mathrm{Pgm}^{\mathrm{b}}$ & - & - & + & 0 & 7 & 70 \\
\hline $\operatorname{Pgm}^{\mathrm{c}}$ & + & + & - & 86 & 73 & 44 \\
\hline $\mathrm{Pgm}^{\mathrm{d}}$ & + & + & + & 66 & 80 & 100 \\
\hline Est $1^{\mathrm{a}}$ & - & - & + & 19 & 42 & 66 \\
\hline Est $1^{b}$ & + & + & + & 100 & 100 & 100 \\
\hline$I d h^{\mathrm{a}}$ & - & + & - & 38 & 58 & 45 \\
\hline $\mathrm{Idh}^{\mathrm{b}}$ & + & + & + & 100 & 100 & 100 \\
\hline $\mathrm{Adh}^{\mathrm{a}}$ &.+ & + & + & 100 & 100 & 100 \\
\hline $\mathrm{Adh}^{\mathrm{b}}$ & + & + & + & 100 & 100 & 100 \\
\hline $\mathrm{Pgia}^{\mathrm{a}}$ & - & - & - & 0 & 2 & 0 \\
\hline $\mathrm{Pgic}^{\mathrm{c}}$ & + & + & + & 100 & 100 & 100 \\
\hline $\mathrm{Pgid}^{\mathrm{d}}$ & + & + & + & 100 & 100 & 100 \\
\hline Lap $^{\mathrm{a}}$ & + & + & + & 87 & 89 & 100 \\
\hline $\mathrm{Lap}^{\mathrm{b}}$ & - & $\ldots$ & + & 0 & 3 & 42 \\
\hline $\mathrm{Lap}^{\mathrm{c}}$ & + & + & + & 100 & 100 & 100 \\
\hline Lap $^{d}$ & - & - & - & 0 & 13 & 0 \\
\hline
\end{tabular}

+ : Présence.

- : Absence.

TABLEAU 6

Classification en 3 groupes "enzymatiques" des "descendances vertes" de "Golden " et "Topred". Division into 3 enzyme classes of the "green progenies" from "Golden" and "Topred".

\begin{tabular}{|c|c|c|c|c|}
\hline & \multicolumn{2}{|c|}{$\begin{array}{c}\text { "Descendance verte" } \\
\text { de Golden }\end{array}$} & \multicolumn{2}{|c|}{$\begin{array}{c}\text { "Descendance verte " } \\
\text { de Topred }\end{array}$} \\
\hline & Effectif & $\%$ & Effectif & $\%$ \\
\hline Groupe 1 & 10 & 23 & 12 & 30 \\
\hline Groupe 2 & 20 & 47 & 23 & 55 \\
\hline Groupe 3 & 13 & 30 & 6 & 15 \\
\hline
\end{tabular}

variabilité importante parmi les descendants à phénotype vert d'un même croisement, tant pour les caractères morphologiques qu'enzymatiques.

Le contrôle monogénique du caractère marqueur (gène R) n'est pas remis en question. Si un déterminisme polygénique était en jeu, le taux d'individus recombinés, à phénotype vert, devrait être constant pour un même croisement. Or, il n'en est rien, ce taux est variable selon les années. La comparaison des descendants rouges et verts issus du croisement "Topred " $x$ “ T.N.R. " apporte d'autres arguments : la distribution de la plupart des caractères observés sur arbres adultes est bien différente parmi les 2 types de descendants. Enfin, les analyses enzymatiques indiquent que 2 allèles, présents chez le parent mâle "T.N.R. » uniquement, ne sont transmis à aucun des descendants verts de "Golden " et seulement à 3 descendants verts de "Topred».

L'intervention de pollen extérieur au croisement est nettement démontrée par l'ensemble des résultats. Les "descendances vertes de Golden » et de «Topred " se caractérisent par leur hétérogénéité supérieure à celles des descendances témoins (hybride et autofécondation), pour la plupart des caractères notés sur arbres adultes. Cette variabilité peut difficilement être expliquée par l'hypothèse d'autofécondation et encore moins par le développement de gamètes non réduits ; en effet, quel que soit le mécanisme en cause, la transmission d'une partie importante du génotype maternel doit assurer en ce cas une relative homogénéité des descendants. Seule l'hypothèse de l'apport de pollen étranger justifie la variabilité importante des « descendances vertes $»$. Les analyses enzymatiques révèlent la présence d'allèles extérieurs au croisement parmi les descendants verts et démontrent ainsi l'intervention de pollen étranger pour tous les individus du groupe 3, soit 30 p. 100 de la " descendance verte de Golden », et 15 p. 100 de celle de «Topred». Cette estimation du taux de pollen étranger est vraisemblablement très inférieure à la réalité. En effet seule l'intervention de pollen porteur d'un allèle absent chez les 2 parents du 
croisement peut être décelée, et les 3 parents, «Golden », « Topred » et «T.N.R. », possèdent ensemble la grande majorité des allèles fréquents parmi les variétés de pommier.

Cet apport de pollen extérieur au croisement peut être attribué aux abeilles employées pour effectuer la pollinisation. Les travaux de KRAII (1962) démontrent, à l'aide de plusieurs gènes marqueurs, que les abeilles ne portent plus de pollen capable de germer après $12 \mathrm{~h}$ de séjour dans la ruche; ce laps de temps était respecté dans notre expérience. Mais ces travaux ont été réalisés sur différentes espèces maraîchères et florales et ne sont peut-être pas transposables au pommier. En 1949, SingH \& BOYNTON ont étudié la capacité de germination du pollen récolté sous forme de pelotes par les abeilles, sur plusieurs variétés de pommier : celle-ci diminue considérablement dès les $1^{\text {res }}$ heures de conservation, puis s'annule. Cependant, ces résultats ne tiennent pas compte du pollen présent sur le corps des abeilles, qui n'a pas subi de dégradation par les substances sécrétées lors de la formation des pelotes. Ce type de pollen peut être à l'origine d'une partie des descendants verts. La variabilité du taux de plantes vertes obtenu selon les croisements peut être reliée à des différences d'activité ou aux types de pommiers visités la veille par les abeilles de chaque ruchette.

L'hypothèse d'autofécondation peut être avancée pour expliquer l'apparition de 2 types de descendants verts. Un taux élevé d'individus triploïdes a été obtenu, en particulier dans la descendance de «Golden ». Les 9 triploïdes issus de «Golden » qui ont pu faire l'objet d'observations sur fruits ont des caractéristiques assez voisines des descendants de $2^{\mathbf{e}}$ génération d'autofécondation de cette même variété. L'analyse enzymatique réalisée sur 8 d'entre eux n'a révélé que des allèles présents chez «Golden ». Les autres individus triploïdes n'ont pas encore atteint le stade de mise à fruit ; la plupart d'entre eux sont de phénotype chétif. Toutes ces observations sont en accord avec l'hypothèse d'autofécondation par des grains de pollen non réduits, un phénomène de compétition entre allèles différents au locus $\mathrm{S}$ d'autoincompatibilité rendant possible la fécondation. La variation du taux de triploïdes selon les croisements correspondrait alors à une aptitude à la formation de gamètes non réduits variable selon les génotypes. L'autofécondation peut également être à l'origine d'un autre groupe d'individus : les descendants diploïdes à phénotype chétif. En effet, leur vigueur réduite est tout à fait comparable à l'effet d' « inbreeding " caractéristique de la descendance de $2^{\mathrm{e}}$ génération d'autofécondation de "Golden». Du fait de l'absence de fleurs et de fruits sur ces individus, ni les observations détaillées ni les analyses enzymatiques n'ont pu être réalisées. La présence de quelques individus aneuploïdes parmi ce groupe reste possible. Cependant la survie des quelques individus à 35 chromosomes détectés au stade jeune semis a été très faible après le passage en pépinière. La proportion d'aneuploïdes parmi les descendants actuellement vivants est donc certainement très faible.
L'hypothèse du développement par parthénogenèse d'oosphères non réduites n'a pu être ni confirmée ni infirmée par nos résultats. Seule une remarque peut être faite : si certains individus sont issus d'un doublement post-méïotique, ils doivent être entièrement homozygotes. Parmi tous les individus ayant fait l'objet d'analyses enzymatiques, seuls 2 descendants verts de «Topred » présentent, pour tous les systèmes étudiés, un phénotype compatible avec un génotype homozygote. D'autre part, le phénotype très chétif de certains individus peut correspondre à une situation d'homozygotie totale.

Tous les individus pour lesquels les résultats indiquent une origine maternelle possible (autofécondation ou développement de gamètes non réduits) sont conservés et feront l'objet d'études complémentaires.

\section{CONCLUSION}

L'observation de caractères morphologiques ou agronomiques et l'analyse enzymatique ont apporté dans cette étude des résultats complémentaires. La mise en évidence de l'apport de pollen étranger dû à l'emploi des abeilles a des conséquences importantes pour tout programme d'études génétiques : s'il n'est pas possible de «contrôler » les ruchettes d'abeilles par une méthode d'isolement, seule la pollinisation manuelle pourra être employée. Parmi les descendants des croisements étudiés, les individus triploïdes ou diploïdes chétifs constituent un matériel original, vraisemblablement issu d'autofécondation. L'hypothèse du développement par parthénogenèse de gamètes non réduits n'a pu être vérifiée au cours de cette étude. La recherche d'un tel phénomène mérite cependant d'être poursuivie ; en effet, si le mécanisme en cause est une restitution à la $1^{\text {re }}$ division (F.D.R.), environ 75 p. 100 du génotype parental est transmis aux descendants sans recombinaison (MOK \& PELOQUIN, 1975b). C'est là une voie de modification d'un génotype intermédiaire entre les 2 méthodes classiques : hybridation ou mutagenèse. Si le mécanisme en cause est un doublement post-méiotique, c'est un moyen d'obtenir directement des individus « haploïdes doublés».

Depuis 1981, une série de croisements a été réalisée dans le prolongement de cette $1^{\text {re }}$ étude. L'adaptation de la technique d'électrophorèse à l'analyse de feuilles permet d'étudier ces descendances avant le stade adulte. Les objectifs poursuivis sont les suivants : quantifier l'apport de pollen étranger par les abeilles et tester une méthode de " contrôle » des ruchettes, augmenter la fréquence d'haploïdes par l'emploi de différents types de pollinisation, rechercher par pollinisation manuelle sur une large gamme de variétés d'éventuels phénomènes de développement par parthénogenèse de gamètes non réduits. Ces résultats seront présentés dans un $2^{\mathrm{e}}$ article.

Reçu le 20 février 1985. Accepté le 11 juillet 1985. 


\section{RÉFÉRENCES BIBLIOGRAPHIQUES}

Chevreau E., 1984. Contribution à l'étude de la génétique du pommier: apport de l'analyse enzymatique. Thèse Doct. Ing., Orsay, $99 \mathrm{p}$.

Chevreau E., Lespinasse Y., Gallet M., 1986. Inheritance of pollen enzymes and polyploid origin of apple (Malus $\times$ domestica Borkh.). Theor. Appl. Genet. (sous presse).

Decourtye L., 1967. Etude de quelques caractères à contrôle génétique simple chez le pommier (Malus sp.) et le poirier (Pyrus communis). Ann. Amélior. Plantes, 17 (3), 243-265.

Einset J., 1952. Spontaneous polyploidy in cultivated apples. $J$. Am. Soc. Hortic. Sci., 59, 291-302.

Fleckinger J., 1964. Le Bon Jardinier, p. 362-372, P. Grisvard \& V. Chaudun, La Maison Rustique, Paris.

Kapusta V., 1983. Levée de l'auto-incompatibilité chez le pommier (Malus pumila Mill.). Etude comparative de clones autocompatibles. Mémoire de fin d'Etudes, E.N.S.A. Rennes.

Kraii A., 1962. How long do honey bees carry germinable pollen on them? Euphytica, 11, 53-56.

Lespinasse Y., 1973. Chromosomic observations in Malus and Pyrus. Proc. Eucarpia Fruit Section Symp. V, Canterbury, 75-83.

Lespinasse Y., Godicheau M., 1980. Création et description d'une plante haploïde de pommier (Malus pumila Mill.). Ann. Amélior. Plantes, 30 (1), 39-44.

Lewis D., Modlibowska I., 1942. Genetical studies in pears IV. Pollen tube growth and incompatibility. J. Genet., 43, 211-222.

Modlibowska I., 1945. Pollen tube growth and embryo-sac development in apples and pears. J. Pomol. Hortic. Sci., 21, 58-87.
Mok D. W. S., Peloquin S. J., 1975a. Three mechanisms of $2 \mathrm{n}$ pollen formation in diploid potatoes. Can. J. Genet. Cytol., 17, 217-225.

Mok D. W. S., Peloquin S. J., 1975b. The inheritance of three mechanisms of diplandroid ( $2 \mathrm{n}$ pollen) formation in diploid potatoes. Heredity, 35 (13), 295-302.

Sax K., 1959. The cytogenetics of facultative apomixis in Malus species. J. Arnold Arbor., 40, 289-297.

Schmidt H., 1977. Contributions on the breeding of apomictic apple stocks. 4. On the inheritance of apomixis. Z. Pflanzenzucht., $78,3-12$.

Sherman W. B., Knight R. J., Lyrene P. M., 1978. Probable apomixis in "Anna", a diploid apple cultivar. Hort. Science, 13 (2), 162.

Singh S., Boynton D., 1949. Viability of apple pollen in pollen pellets of honey bees. J. Am. Soc. Hortic. Sci., 53, 148-152.

Sokal R. R., Rohlf F. H., 1969. Biometry. The principles and practice of statistics in biological research. W. H. Freeman et al., San Francisco, p. 559-560.

Taylor L. M., 1978. Variation patterns of parthenogenetic plants derived from "unreduced" embryo-sac of Solanum tuberosum species andigena (Juz. et Buck.) Hankes. Theor. Appl. Genet., 52 , 241-249.

Zwintscher M., 1973. Malus pumila var. niedzwetzkyana and its role in apple breeding. Proc. Eucarpia Fruit Section Symp. V., Canterbury, 154-162. 